\title{
A Newly Designed EGFP-2A Peptide Monocistronic Baculoviral Vector for Concatenating the Expression of Recombinant Proteins in Insect Cells
}

\author{
Chih-Yu Wu ${ }^{1,2, *,+}$, Chao-Wei Huang ${ }^{3,+}{ }^{\circ}$, Yu-Shin Nai ${ }^{4,+}+{ }^{\circ}$, Pei-Yu Chu ${ }^{5}$, \\ Chung-Hsiung Wang ${ }^{6, *}$ and Shih-Torng Ding ${ }^{1,3, *}$ \\ 1 Center of Biotechnology, National Taiwan University, Taipei 10617, Taiwan \\ Department of Chemical Engineering, National Taiwan University, Taipei 10617, Taiwan \\ 3 Department of Animal Science and Technology, National Taiwan University, Taipei 10617, Taiwan; \\ d98626004@ntu.edu.tw \\ 4 Department of Entomology, National Chung Hsing University, Taichung 402, Taiwan; ysnai@nchu.edu.tw \\ 5 Department of Plant Pathology and Microbiology, National Taiwan University, Taipei 10617, Taiwan; \\ r93445108@ntu.edu.tw \\ 6 Department and Graduate Institute of Entomology, National Taiwan University, Taipei 10617, Taiwan \\ * Correspondence: picorna.tw@yahoo.com.tw (C.-Y.W.); wangch@ccms.ntu.edu.tw (C.-H.W.); \\ sding@ntu.edu.tw (S.-T.D.); Tel.: +886-2-33661454 (C.-Y.W.) \\ + These authors contributed equally to this work.
}

Received: 29 March 2019; Accepted: 23 April 2019; Published: 15 May 2019

\begin{abstract}
Recombinant proteins produced by the baculovirus expression vector system (BVES) have been widely applied in the agricultural and medical fields. However, the procedure for protein expression is inefficient and needs to be improved. Herein, we propose a simple construct that incorporates a selectable marker (enhanced green fluorescent protein, EGFP) and a picorna viral-derived "self-cleaving" 2A-like peptide to separate the EGFP and target proteins in a monocistronic baculovirus vector to facilitate isolation of the recombinant baculovirus in the BVES. In this study, porcine adiponectin (ADN), a secreted, multimeric protein with insulin-sensitizing properties, was used to demonstrate its utility in our EGFP-2A-based expression system. EGFP and ADN were simultaneously expressed by a recombinant alphabaculovirus. Co-expression of EGFP facilitates the manipulation of the following processes, such as determining expression kinetics and harvesting ADN. The results showed that the 2A "self-cleaving" process does not interfere with EGFP activity or with signal peptide removal and the secretion of recombinant ADN. Posttranslational modifications, including glycosylation, of the recombinant ADN occurred in insect cells, and the formation of various multimers was further verified. Most importantly, the insect-produced ADN showed a similar bioactivity to that of mammalian cells. This concept provides a practical and economic approach that utilizes a new combination of alphabaculovirus/insect cell expression systems for future applications.
\end{abstract}

Keywords: baculovirus expression system; 2A-mediated "cleavage" process; recombinant porcine adiponectin; multimer formation; N-linked glycosylation

\section{Introduction}

Baculoviruses are known to infect over 660 insect host species. The availability of lepidopteran cell lines has steadily increased at about 50 new lines per decade, and more than 30 baculoviruses that have been grown in insect cell cultures have been reported [1]. Over the last 30 years, recombinant baculoviruses have been widely used to express foreign proteins in insect cells because of their 
capacity for large inserts of heterologous genes and a high yield of recombinant protein [2]. Proteins produced in insect cells are very similar to naturally occurring mammalian proteins in terms of posttranslational modifications, biological activity, and protein stability [3,4]. Unfortunately, the current commercial baculovirus expression vector systems (BEVSs), mostly Autographa californica multiple nucleopolyhedrovirus (AcMNPV)-based systems, have two major limitations in comparison to other higher eukaryotic expression systems. First, cells used in the BEVS cannot produce complex-type N-glycosylated proteins. Second, cells lyse and die quickly in response to viral infection, and thus, the cell secretory pathway is impaired during the late stage of viral infection, and recombinant protein production is typically restricted to three days [5]. However, we doubt these limitations are universal in all kinds of baculovirus/insect cell interactions. As we know, baculoviruses are very diverse in terms of gene content and genome organization. For example, an alphabaculovirus (lepidopteran-specific nucleopolyhedrovirus (NPV), such as Autographa californica multiple nucleopolyhedrovirus (AcMNPV) has about 150 genes but only shares 38 core genes with other alphabaculoviruses [6]. This means that over 100 genes in an alphabaculovirus are specific and may uniquely interact with their native host insect cells. We therefore expect that establishing a new combination of baculovirus/insect cell expression systems may break through the limitations of the current BEVSs.

During manipulation of the current BEVSs, or establishing a new system for protein expression, several barriers (e.g., inefficiency of isolating a recombinant baculovirus vector, long processing time, difficulty visualizing plaques or recognizing the cytopathogenic effect of infected cells) may occur, especially when using a polyhedron-negative phenotype as a genetic marker for positive recombinants. Although these problems may be solved by introducing the galactosidase $\beta$-(gal) gene (lacZ) into the transfer vectors, this increases the cost of the selective process due to the need for an additional substrate, 5-bromo-4-chloro-3-indolyl- $\beta$-D-galactopyranoside (X-Gal), for this marker [7]. Incorporating the enhanced green fluorescent protein (EGFP) gene instead of lacZ into the baculovirus creates easy visualization of the gene expression, while a multiple expression system will be required to express the EGFP and recombinant protein of interest simultaneously. We therefore hypothesize that co-expression of the EGFP and target protein via fusion with a "self-cleaving" 2 A peptide [8,9] would allow one to utilize a new combination of baculovirus/insect cell expression systems. To date, many successful examples of using a 2A peptide-based baculovirus vector for the expression of dual or multiple proteins in insect and other animal cells [10-14] have further supported our ideas.

This study attempts to demonstrate a multiple protein expression system by concatenating a selectable marker and a viral-derived "self-cleaving" $2 \mathrm{~A}$ peptide in a monocistronic baculovirus vector to facilitate the utilization of a baculovirus/insect cell expression system (Figure 1). A 2A-like peptide element derived from a picornavirus, Perina nuda virus (Genus: Iflavirus), and an alphabaculovirus, Lymantria xylina multiple NPV (LyxyMNPV), and its homolog cell system were chosen to demonstrate the accessibility of our concept. In this system, two concatenated proteins, EGFP and full-length porcine adiponectin (ADN), will test the "self-cleaving" ability of a 2A-like peptide element. Co-expression of EGFP is expected to facilitate the detection and isolation of recombinant proteins and viruses, determinations of viral titer, protein expression kinetics, and harvest time. Moreover, the posttranslational modifications of the recombinant ADN were verified in our expression system. Based on our data, the recombinant ADN produced by our system revealed similar bioactivity to that of mammalian cells. Although the studies are in their infancy, cell death and lysis were delayed in our system compared to a conventional AcMNPV system, which may improve the integrity and quality of recombinant proteins. 


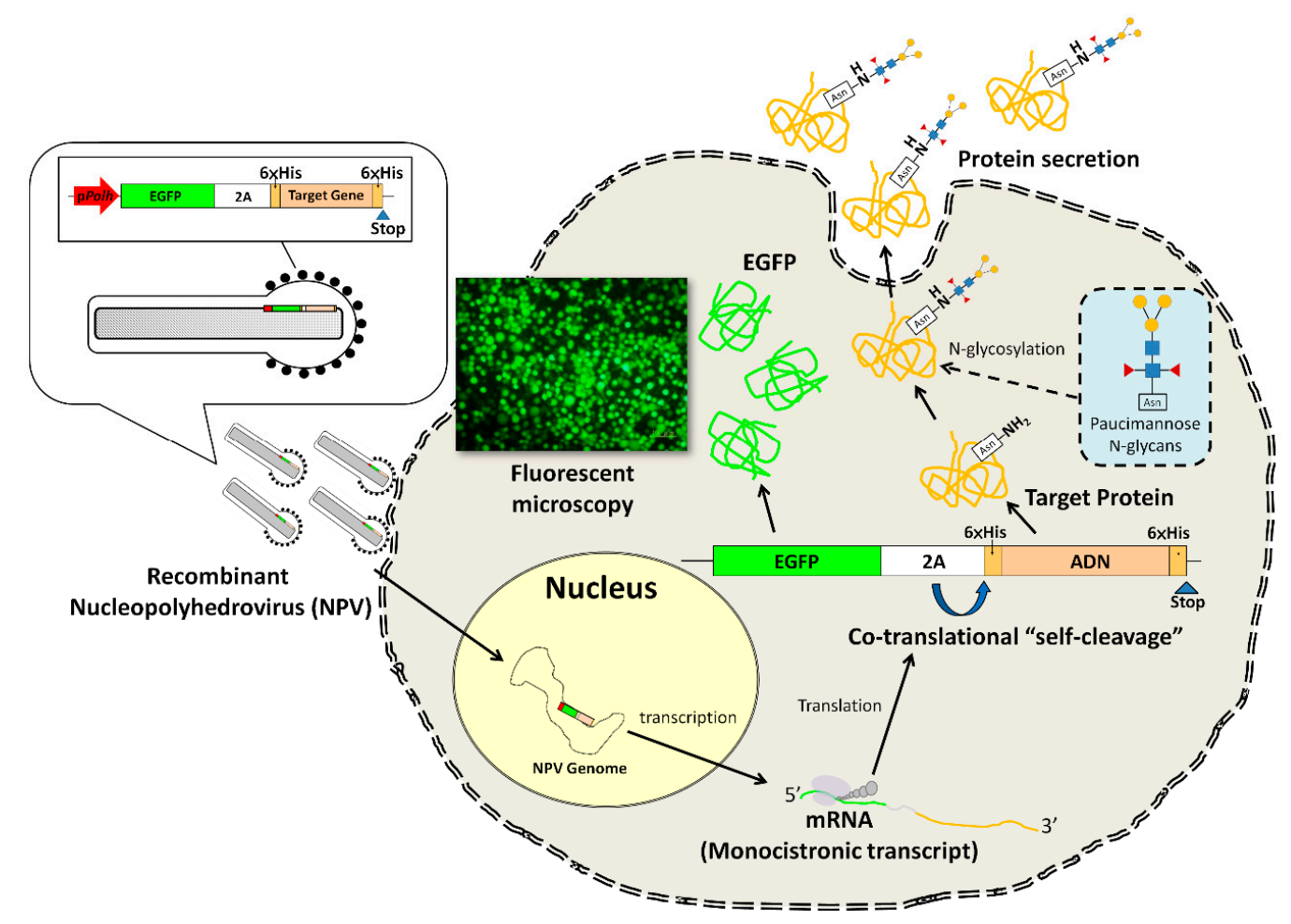

Figure 1. Facile protein expression system concept. A facile construct incorporating a selectable marker and a viral-derived "self-cleaving" 2A peptide in a monocistronic baculovirus vector to facilitate the utilization of a new baculovirus-insect cell expression system based on unutilized viral species is proposed. The 2A-like peptide element derived from Perina nuda virus mediates "self-cleaving" in Lymantria xylina NTU-LY cells and thus separates two proteins, enhanced green fluorescent protein (EGFP) and full-length porcine adiponectin (ADN), simultaneously expressed by a recombinant L. xylina multiple nucleopolyhedrovirus. Co-expression of EGFP facilitates the efficiency of ADN production by the easy detection and isolation of recombinant viruses and determination of expression kinetics and harvest time. Posttranslational modifications of the recombinant ADN, including N-linked glycosylation and various multimer formation, then further occur in NTU-LY insect cells. The 2A "self-cleaving" process does not interfere with EGFP activity or signal peptide removal and the secretion of recombinant ADN. The secreted recombinant ADN can be easily collected and purified by immobilized metal affinity chromatography (IMAC).

\section{Materials and Methods}

\subsection{Cell lines and Virus Growth}

The Lymantria xylina cell line, NTU-LY [15], was maintained at $28^{\circ} \mathrm{C}$ in TNM-FH insect medium (Sigma-Aldrich, USA) plus supplements [16]. The supplements contained $50 \mathrm{IU} \mathrm{ml}^{-1}$ penicillin, $50 \mu \mathrm{g} \mathrm{ml}^{-1}$ streptomycin, $1.25 \mathrm{mg} \mathrm{ml}^{-1}$ amphotericin B (Sigma-Aldrich, USA), and 8\% fetal bovine serum (FBS, Hyclone). For foreign protein expression, the NTU-LY cells were adapted to and grown in a commercial Sf900II-SFM (Invitrogen, California, USA). NTU-LY monolayers were used for the propagation of the wild-type $(\mathrm{Wt})$ clone of LyxyMNPV, LyxyMNPV-Ly5, and recombinant viruses as per a previously reported study $[15,17]$. The human hepatic adenocarcinoma cell line, SK-Hep1, was cultured in Dubecco's modified eagle medium (DMEM) supplemented with 2 mM L-glutamine, $0.1 \mathrm{mM}$ non-essential amino acids (NEAA), $1.0 \mathrm{mM}$ sodium pyruvate, and $10 \% \mathrm{FBS}$ in an incubator with $5 \% \mathrm{CO}_{2}$ at $37^{\circ} \mathrm{C}$. 


\subsection{Construction of Transfer Vectors}

The EGFP-2A-based monocistronic baculovirus transfer vectors, pLxGFP-2A-ADN and pLxGFP-m2A-ADN (Figure 2), were constructed by standard cloning procedures. The primers used for the polymerase chain reaction (PCR) are listed in Table 1. Briefly, a baculovirus transfer vector, pLyxyExp, was produced by inserting the flanking regions either side of the LyxyMNPV polyhedrin gene (polh) into the pBlueScript II SK (-) (pBS.SK-, Stratagene) vector. First, the pUC18 vector (Roche, Basel, Switzerland) was digested with SmaI, dephosphorylated with calf intestinal alkaline phosphatase (Roche, Basel, Switzerland), and ligated with an EcoRV-K fragment from the LyxyMNPV genome (GenBank ID: AY842284), in which the polh is located, to produce pLxRV-K. Then, a 2.2-kbp upstream region of the polh was generated by the PCR amplification of pLxRV-K using the M13 forward primer M13-F and the reverse primer polh-up(R). This PCR-amplified fragment was then cloned into the pGEM-T easy vector (Promega, Wisconsin, USA), retrieved by digestion with BamHI and EcoRV, and inserted into corresponding enzyme digested pBS.SK- to produce an intermediate plasmid pBS.SK-/up. Furthermore, a 1.8-kbp downstream region of the polh was amplified from pLxRV-K with the following two additional oligonucleotides: Forward primer polh-down (F) and the M13 reverse primer M13-R. The PCR product was cloned, retrieved by digestion with HindIII and KpnI, and then inserted into corresponding enzyme digested pBS.SK-/up to form the vector pLyxyExp. The pLyxyExp vector was further modified by eliminating unwanted restriction enzyme sites, including a BamHI-SacII fragment and a KpnI site. This was accomplished by digesting the pLyxyExp vector with the corresponding restriction enzymes, treatment of the restriction sites with T4 DNA polymerase (Promega, Wisconsin, USA), and religation of the free ends of the vector. Subsequently, a polylinker containing recognition sites for EcoRV, KpnI, NheI, SmaI, PstI, SacII, and HindIII was synthesized using the complementary oligonucleotides MCS-F and MCS-R, which were annealed to yield a double-stranded fragment and then cloned into the modified pLyxyExp vector after digestion with EcoRV and HindIII. The resulting construct harboring a multiple cloning site which allows for the cloning of a target gene with expression under the control of the very late polh promoter was named pLyxyExp-MCS (Figure 2). The target gene encoding a fusion protein, which was composed of an EGFP, a 2A peptide, and a porcine adiponectin $(\mathrm{ADN})$, was cloned into the vector pLyxyExp-MCS. Briefly, EGFP was PCR-amplified from pEGFP-C1 vector (Clontech, California, USA) using one GFP-forward primer and two different GFP-reverse primers, one encoding the normal 2A peptide from Perina nuda virus and the other encoding a noncleavable mutant $2 \mathrm{~A}$ peptide (referred to here as $\mathrm{m} 2 \mathrm{~A}$ ). Both reverse primers omitted the EGFP stop codon, so that translation could continue into the 2A peptide and beyond. Next, ADN was PCR-amplified from the previously cloned full-length cDNA of porcine ADN (Ding et al. 2004) using the primers EcoRI/His-ADN_F and SacII/His-ADN_R. The amplified gene fragments encoding EGFP-2A (or EGFP-m2A) and 6x Histidine-tagged ADN were digested with EcoRI and then ligated together with T4 DNA ligase (Promega, Wisconsin, USA). Two resulting constructs, EGFP-2A-ADN and EGFP-m2A-ADN, served as templates for the PCR amplification using the primers GFP-forward and SacII/His-ADN_R. The PCR product for these two constructs was treated with XbaI and SacII and cloned into the pLyxyExp-MCS vector, and the resulting plasmids were called pLxEGFP-2A-ADN and pLxEGFP-m2A-ADN. Sequences of pLxEGFP-2A-ADN and pLxEGFP-m2A-ADN were confirmed by DNA sequencing with at least a two-fold overlap. 
Table 1. The primers used in this study.

\begin{tabular}{|c|c|c|}
\hline Name & Sequence & Note \\
\hline M13-F & 5'-GTTGTAAAACGACGGCCAGT-3' & - \\
\hline M13-R & 5'-CAGGAAACAGCTATGACC-3' & - \\
\hline polh-up(R) & 5'-CCATGATATCTTTACGGTGTATTTGATTTTTCCAAAATCTTTACG-3' & $\begin{array}{l}\text { corresponding to } 1977-2011 \mathrm{nt} \text { in the } \\
\text { LyxyMNPV EcoRV-K fragment; the } \\
\text { EcoR V site is underlined }\end{array}$ \\
\hline polh-down $(\mathrm{F})$ & 5'-GGTTAAGCTTACGATTACGCCTCGGATTCC-3' & $\begin{array}{l}\text { corresponding to } 2753-27 / 2 \text { nt in the } \\
\text { LyxyMNPV EcoR V-K fragment; the } \\
\text { HindIII site is underlined }\end{array}$ \\
\hline MCS-F & 5'-ATCGGTACCGCTAGCTCTAGACCCGGGCTGCAGCCGCGGA-3' & - \\
\hline MCS-R & 5'-AGCTTCCGCGGCTGCAGCCCGGGTCTAGAGCTAGCGGTACCGAT-3' & - \\
\hline GFP-reverse primer_1 & $\begin{array}{c}\text { 5'-GCGCGAATTCGGGCCCAGGATTCGA } \\
\text { CTCGATGTCACCATCTTGTGTCAAATCCTTCTGCCCACCACCAATAATATTCTTGTACAGCTCGTCCATGCC-3' }\end{array}$ & $\begin{array}{l}\text { encoding the normal 2A peptide } \\
\text { from Perina nuda virus; the EcoRI site } \\
\text { is underlined }\end{array}$ \\
\hline GFP-reverse primer_2 & $\frac{5^{\prime} \text {-GCGCGAATTCAGGATTCGACTCGAT GTCACCATCTTGTGTCAAATCCTTCTG }}{\text { CCCACCACCAATAATATTCTTGTACAGCTCGTCCATGCC-3' }}$ & $\begin{array}{l}\text { encoding a noncleavable mutant } 2 \mathrm{~A} \\
\text { peptide; the EcoRI site is underlined }\end{array}$ \\
\hline EcoRI/His-ADN_F & 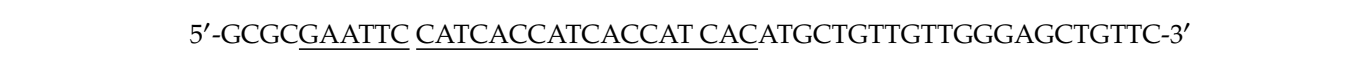 & $\begin{array}{l}\text { the sequences for the EcoRI site and } \\
6 \times \text { Histidine tag are underlined }\end{array}$ \\
\hline SacII/His-ADN_R & 5'-CGCGCCGCGGTCAGTGATGGTGATG GTGATGTTCAATGTTGTGGTAGAGAAG GAAG-3' & $\begin{array}{l}\text { the sequences for the SacII site and } 6 x \\
\text { Histidine tag are underlined }\end{array}$ \\
\hline
\end{tabular}




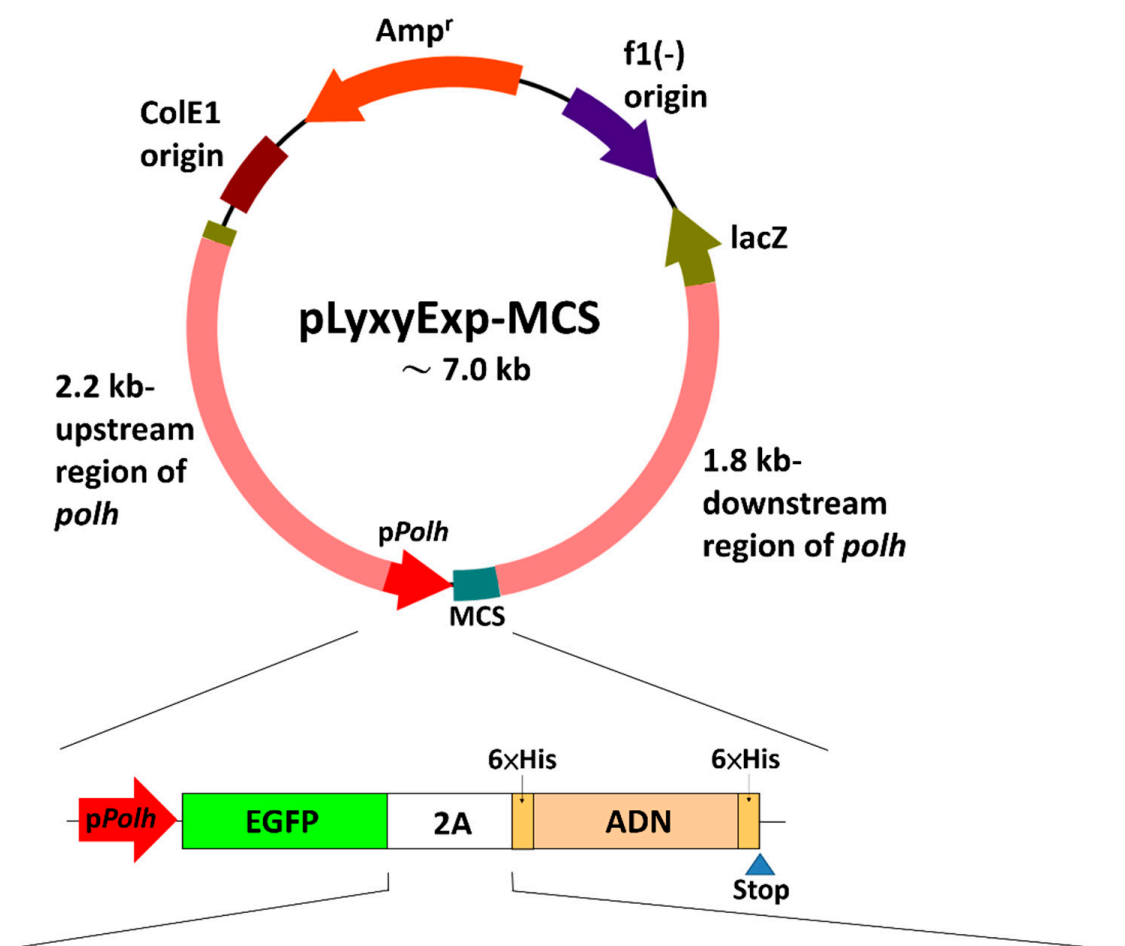

pLxEGFP- AAT ATT ATT GGT GGT GGG CAG AAG GAT TTG ACA CAA CAT GGT GAC ATC GAG TCG AAT CCT GGG CCC 2A-ADN
(Functional 2A)

PLXEGFP- AAT ATT ATT GGT GGT GGG CAG AAG GAT TTG ACA CAA CAT GGT GAC ATC GAG TCG AAT CCT GAA TTC

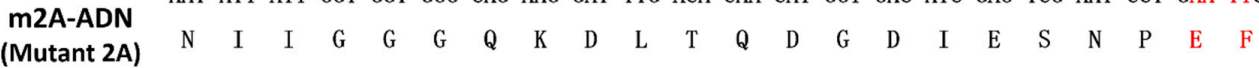

Figure 2. Schematic representation of the pLyxyExp-MCS vector and monocistronic plasmid construction for the co-expression of enhanced green fluorescent protein (EGFP) with porcine adiponectin $(\mathrm{ADN})$ by the baculovirus LyxyMNPV. To test the function of the 2A linker, genes for EGFP and ADN were in-frame fused with the nucleotide sequences encoding either a functional 2A peptide (upper) or a nonfunctional $\mathrm{m} 2 \mathrm{~A}$ peptide (lower). The specific nucleotide and amino acid changes in $\mathrm{m} 2 \mathrm{~A}$ are shown in red. The constructs EGFP-2A-ADN and EGFP-m2A-ADN were then inserted into the pLyxyExp-MCS vector. The resultant plasmids were designated pLxEGFP-2A-ADN and pLxEGFP-m2A-ADN, respectively.

\subsection{Recombinant Virus Generation and Cell Viability Analysis}

The plasmids, pLxEGFP-2A-ADN and pLxEGFP-m2A-ADN, were transfected into NTU-LY cells $\left(1 \mu \mathrm{g}\right.$ of plasmid DNA was transfected into $3 \times 10^{5}$ cells per well in Nunc ${ }^{\mathrm{TM}} 4$-Well Dishes) using Cellfectin reagent (Invitrogen, California, USA) in accordance with the manufacturer's protocol. After gentle shaking at room temperature for $5 \mathrm{~h}$, the transfection mixture was removed and replaced with fresh TNM-FH medium. Then, the cells were inoculated with wt-LyxyMNPV at a multiplicity of infection (MOI) of 10 and incubated at $28{ }^{\circ} \mathrm{C}$. Monitoring was done for $7 \mathrm{~d}$ posttransfection to ascertain successful expression of the recombinant virus via the green fluorescent plaques observed by fluorescence microscopy (Olympus IX71). The recombinant virus was subsequently purified by three rounds of plaque screening following the protocols described by Summer and Smith (Summers and Smith 1987). The resulting viruses were named vLxEGFP-2A-ADN and vLxEGFP-m2A-ADN. The virus titer was measured using the end-point dilution method (TCID 50 analysis) (Reed and Muench 1938) by EGFP fluorescence detection in a 96-well plate. For RT-PCR analysis, two clones of each recombinant virus were picked from the 96-well plate at a dilution of $10^{-5}$ and designated vLxEGFP-2A-ADN_5C, vLxEGFP-2A-ADN_5G, vLxEGFP-m2A-ADN_5B, and vLxEGFP-m2A-ADN_5D. NTU-LY cells were infected with four recombinant clones at an MOI of 1 and incubated at $28^{\circ} \mathrm{C}$. Total RNA was extracted 
from the infected cells at $72 \mathrm{~h}$ post-infection (p.i.) using Trizol reagent (Invitrogen, California, USA) in accordance with the manufacturer's protocol. A portion of the total RNA sample $(2 \mu \mathrm{g})$ was treated with DNase I (1 unit) in a $10 \mu \mathrm{l}$ reaction containing $1 \times$ DNase I reaction buffer at $37^{\circ} \mathrm{C}$ for $30 \mathrm{~min}$, and the reaction was stopped by adding $1 \mu \mathrm{l}$ of $25 \mathrm{mM}$ EDTA solution, followed by heating the mixture at $75^{\circ} \mathrm{C}$ for $15 \mathrm{~min}$. Mock DNase I-treated samples were prepared and processed in parallel by replacing DNase I with water. One hundred nanograms of DNase I-treated or mock-treated total RNA was used in either one-step RT-PCR or PCR alone (without RT treatment) using the primers GFP-forward and SacII/His-ADN_R. The resulting RT-PCR products were then purified and analyzed by DNA sequencing. For cell viability analysis, NTU-LY cells were infected with vLxEGFP-2A-ADN at an MOI of 5 in triplicate. On days $0-10$ post-infection, the percentage of viable cells was determined by trypan blue (Thermo Fisher Scientific, USA) staining.

\subsection{Expression and Purification of Recombinant Adiponectin}

NTU-LY cells were infected with recombinant viruses (vLxEGFP-2A-ADN and vLxEGFP-m2A-ADN) at an MOI of 1 and incubated at $28{ }^{\circ} \mathrm{C}$ for seven days. Purification of the recombinant $6 \times$ histidine-tagged porcine ADN gene was achieved by immobilized metal affinity chromatography (IMAC) according to the QIAexpressionist protocol (Qiagen, Venlo, Netherlands). Briefly, the soluble fraction of the cell lysate was applied to an nickel-charged affinity resin (Ni-NTA) column. After thoroughly washing, the bound ADN was eluted stepwise with imidazole. To optimize adiponectin production, a time-course analysis of adiponectin and EGFP expression in the culture medium was performed. Briefly, about $2 \times 10^{5} \mathrm{NTU}-\mathrm{LY}$ cells/well were seeded on a 24 -well plate and infected with vLxEGFP-2A-ADN at an MOI of 1 . For the analysis of cumulative expression, mediums were collected from different wells at 0 to 9 days post-infection (day zero was the time at which the inoculum was added). For the analysis of individual (daily) expression, mediums were collected from the same wells at 0-9 days post-infection; however, the wells were refilled with fresh medium after the old medium was collected. To examine the recombinant protein expression and the 2A peptide function, each fraction from the purification process and the collected mediums were subjected to Western blot analysis with anti-GFP (Clontech, California, USA) and anti-His Tagged protein antibodies (Bioman Scientific Co., LTD, Taiwan) (Supplementary file). Moreover, the secreted ADN was purified from cell culture medium using IMAC. Briefly, serum-free culture supernatants were harvested from infected cultures before significant cell lysis occurred. Every $250 \mathrm{~mL}$ supernatant was mixed with $1 \mathrm{~mL}$ $50 \% \mathrm{Ni}-\mathrm{NTA}$ resin and incubated with gentle agitation overnight at $4{ }^{\circ} \mathrm{C}$. The resin was transferred into an empty column, and the bound ADN was eluted with imidazole after thorough washing. The eluate was concentrated by Amicon Ultra-0.5 Centrifugal Filter Units (MWco $=3 \mathrm{kDa}$, Millipore) and dialyzed three times with phosphate buffered saline (PBS, pH 7.4). The purified ADN was stored at $-20{ }^{\circ} \mathrm{C}$ for the following experiments. To clarify the glycosylation of insect cell-expressed ADN, the infected cultures at 2-d post-inoculation were treated with $1 \mu \mathrm{g} \cdot \mathrm{ml}^{-1}$ tunicamycin (N-glycosylation inhibitor). After incubation for $72 \mathrm{~h}$, conditioned medium was collected for Western blot analysis (Supplementary file) with anti-His Tagged protein antibodies (Bioman Scientific Co., LTD, Taiwan), and three replicates were performed. The immunoblots of N-glycosylation of insect cell-expressed ADN were quantified by densitometry, and the statistical analysis was determined by the Student's t-test. For multimerization analysis, the purified ADN was subjected to SDS-PAGE with or without $95{ }^{\circ} \mathrm{C}$ heat denaturation for 10 $\mathrm{min}$, and with or without reduction (treated with $\beta$-ME) [18]. Adiponectin was detected with anti-His Tagged protein (Bioman Scientific Co., LTD, Taiwan) or anti-adiponectin antibodies (from our lab; Supplementary file) by Western blot analysis (Supplementary file). 


\subsection{Phosphorylation of AMPK and ACC by Adiponectin}

The AMPK pathway was analyzed following the protocols from Waki et al. (2003) [18]. Briefly, human liver adenocarcinoma cells (SK-Hep1 cells) from the American Type Culture Collection (ATCC) were incubated in serum-free DMEM medium for $6 \mathrm{~h}$, and then complete medium containing recombinant $\mathrm{ADN}$ was added to the well and incubated for $5 \mathrm{~min}$ at $37^{\circ} \mathrm{C}$. Human ADN (a.a. 16-224) forming high molecular weight (HMW) and hexameric species purchased from Enzo Life Sciences (\#ALX-522-063-C050) was used as a positive control. The reaction was stopped with liquid nitrogen, and the cells were lysed and homogenized with a sonicator in lysis buffer $(25 \mathrm{mM}$ Tris- $\mathrm{HCl}, \mathrm{pH}$ 7.4, $10 \mathrm{mM}$ Na3VO4, $10 \mathrm{mM}$ sodium pyrophosphate, $100 \mathrm{mM} \mathrm{NaF}, 10 \mathrm{mM}$ EDTA, $10 \mathrm{mM}$ EGTA, $1 \mathrm{mM}$ phenylmethylsulfonyl fluoride, $1 \%$ Nonidet P-40). The lysate was centrifuged, and the protein concentration was assayed using the Bradford protein assay reagent (Bio-Rad, California, USA). The same amount of lysate protein was applied to SDS-PAGE under reducing and heat-denaturing conditions, blotted onto PVDF membranes, and immunostained with anti-AMPK, anti-ACC, anti-phosphorylated AMP-activated protein kinase (AMPK) antibodies (Cell Signaling Technology, Massachusetts, USA) or anti-phosphorylated ACC (Cell Signaling Technology) antibodies, $\beta$-actin (Santa Cruz biotechnology, Texas, USA), and anti-adiponectin. The percentage of AMPK and ACC phosphorylation was quantified by Image J.

\section{Results}

\subsection{Recombinant Virus Production and Cell Viability Analysis}

In the present study, an EGFP-2A-based monocistronic baculovirus vector/ NTU-LY insect cell expression system was successfully generated for simultaneous expression of the target protein and EGFP. Due to the emission of green fluorescence, the recombinant viruses, vLxEGFP-2A-ADN and vLxEGFP-m2A-ADN, were easily identified after the infection of the NTU-LY cells (Figure 3A). To verify the expression of EGFP-2A-ADN and EGFP-m2A-ADN transcripts from the baculovirus polh promoter, one-step RT-PCR and PCR was performed with the RNAs with DNase I treatment, using the primers GFP-forward and SacII/His-ADN_R (Figure 3B). When a DNase I-treated total RNA preparation from NTU-LY cells was used as a template, the products of EGFP-2A-ADN and EGFP-m2A-ADN were detected by one-step RT-PCR but not by PCR alone (without RT treatment), confirming the expression of the EGFP-2A-ADN and EGFP-m2A-ADN transcripts. These viral transcripts were then cloned and sequenced, and the sequencing data were consistent with those of our constructs, as shown in Figures 2 and 3. Interestingly, the new expression system has a long-term expression capacity. About $25 \%$ cells are still available, even at $9 \mathrm{dpi}$, while lytic or apoptotic cells are found at $14 \mathrm{dpi}$ (Figure 3C). Cell viability analysis shows that the infected cells display delayed death and lysis in the new system compared to a conventional AcMNPV system, which has only 5 to $10 \%$ living cells at 5 dpi (Figure 3D). The new expression system has a higher cell viability compared with the conventional BEVS, and thus, the secretory protein yield and integrity could be increased. 
A

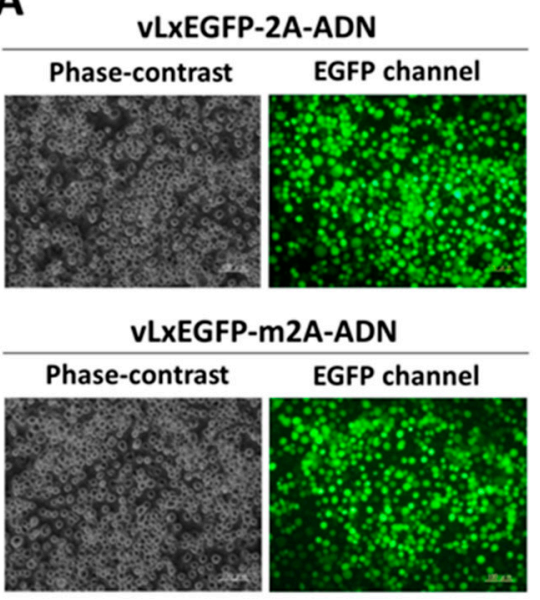

B
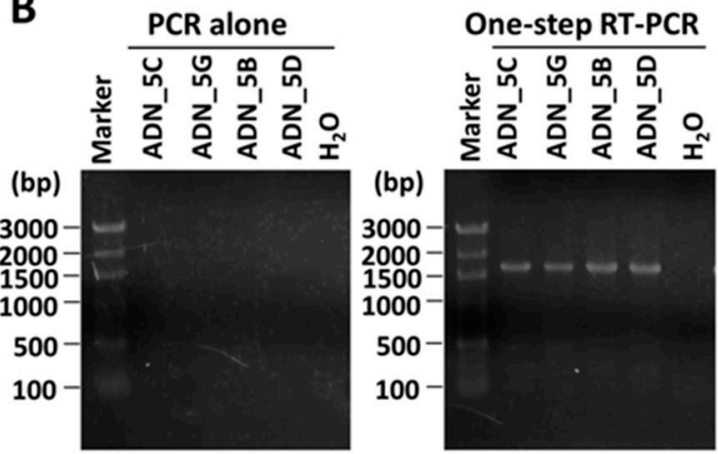

C
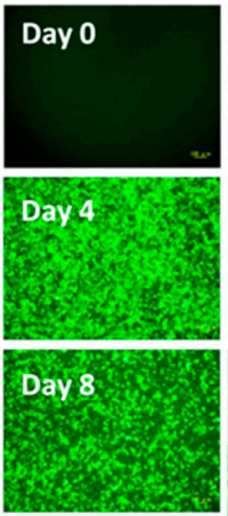
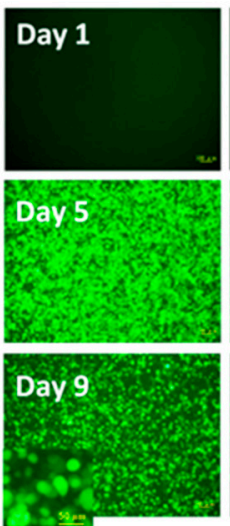
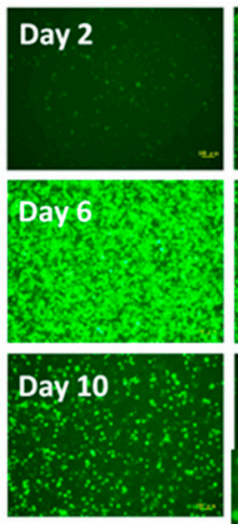
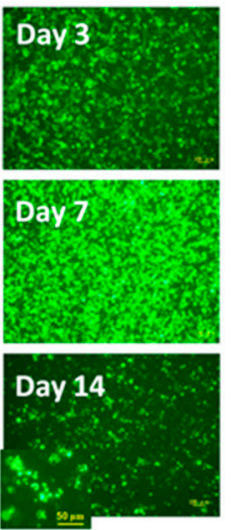

D

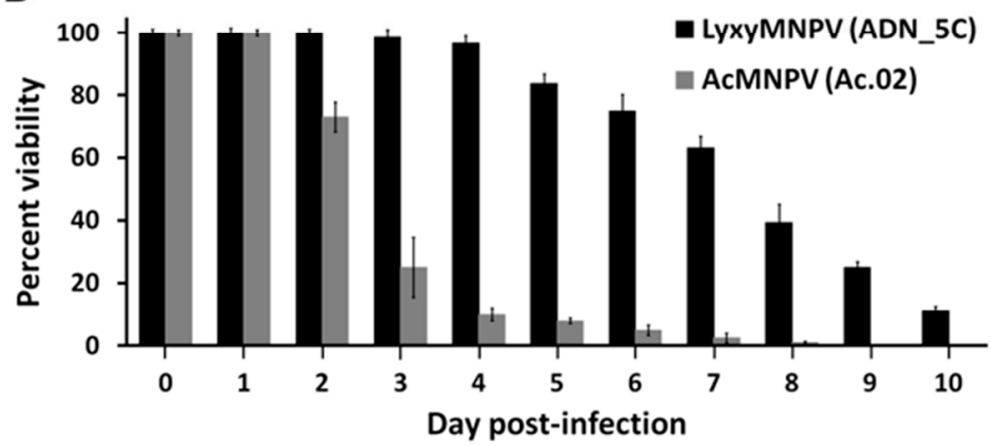

Figure 3. Recombinant virus production and cell viability analysis. (A) The recombinant viruses, vLxEGFP-2A-ADN and vLxEGFP-m2A-and, were generated and propagated in NTU-LY cells. The cells were inoculated with recombinant viruses at a multiplicity of infection (MOI) of 1 and incubated at $28^{\circ} \mathrm{C}$. The expression of EGFP was used to monitor the viral infection in NTU-LY cells. Photographs were taken in the same field under phase-contrast microscopy, or with a GFP filter unit (Excitation: $457-487 \mathrm{~nm}$, Emission: 502-538 nm) by fluorescence microscopy. Scale bar, $100 \mu \mathrm{m}$. (B) Reverse transcriptionpolymerase chain reaction (RT-PCR) analysis of EGFP-2A-ADN and EGFP-m2A-ADN transcripts. PCR (left) and one-step RT-PCR (right) were used to analyze total RNA from cell cultures infected with one of four viral clones indicated at the top. ADN_5C and ADN_5G represent vLxEGFP-2A-ADN clones; ADN_5B and ADN_5D represent vLxEGFP-m2A-ADN clones. All samples were treated with DNase I $(+)$. Sizes (bp) of marker DNAs are indicated on the left. (C) Fluorescent images and (D) cell viability for NTU-LY cells infected with vLxEGFP-2A-ADN at a MOI of 5 are shown for days 1-10 post-infection. Local enlarged images are presented to show the cell integrity. A conventional AcMNPV expression system is used for comparison (data from the literature [5]). 


\subsection{Verification of 2 A Peptide Function}

As Figure 4 shows, the 2A function in the expression system was verified by Western blotting with anti-GFP and anti-His Tagged protein antibodies. Specifically, the blots for vLxEGFP-2A-ADN contained EGFP (Band 4), the EGFP-2A fragment (Band 3), and ADN fragments (Band c and d), indicating that $v$ LxEGFP-2A-ADN has a functional 2A peptide that mediated an effective cleavage between 2A and ADN. By contrast, the blots for vLxEGFP-m2A-ADN contained EGFP (Band 4), EGFP-2A-ADN fragments (Band 1 or a, and Band 2), and 2A-ADN fragment (Band b), indicating that vLxEGFP-m2A-ADN has a nonfunctional 2A peptide (Figure 4A). The molecular weights of these bands shown in parenthesis were calculated according to their amino acid sequences. Bands 1, 2, 3, 4 and $a, b, c, d$ can be recognized by anti-GFP or anti-His antibodies, due to the fact that they contain an EGFP protein and/or C-terminal His-tag. Based on the design, the expected products should be band 1 or band a, band 3 , band $\mathrm{c}$, and band $\mathrm{d}$, while band 2 , band 4 , and band $\mathrm{b}$ are putative products (Figure 4B). The protease cleavage-generated EGFP protein (Band 4) in both soluble and flow-through fractions is shown in blots detected with anti-GFP antibodies. Notably, the major purified protein, ADN-His (Band d), for vLxEGFP-2A-ADN was slightly smaller than the fragment of His-ADN-His (Band c), suggesting the possibility that the recombinant ADN produced by NTU-LY cells may undergo signal peptide removal. Based on our investigations, a signal peptide predicted at the $\mathrm{N}$-terminal sequence of full-length ADN using SignalP 4.0 website software [19] and a matched molecular weight of protein found in culture medium (see below) could partially support the suggestion. However, more evidence needs to be provided for proof of this speculation (e.g., mass spectrum sequencing data). Several low-molecular bands (Figure 4A, arrowheads) that supposedly resulted from protein degradation were also observed in blots, while the identities of these bands were beyond the scope of this paper. 
A

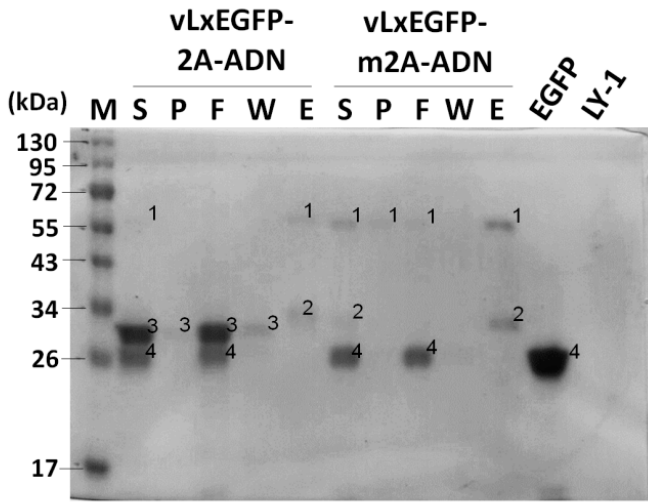

Anti-GFP

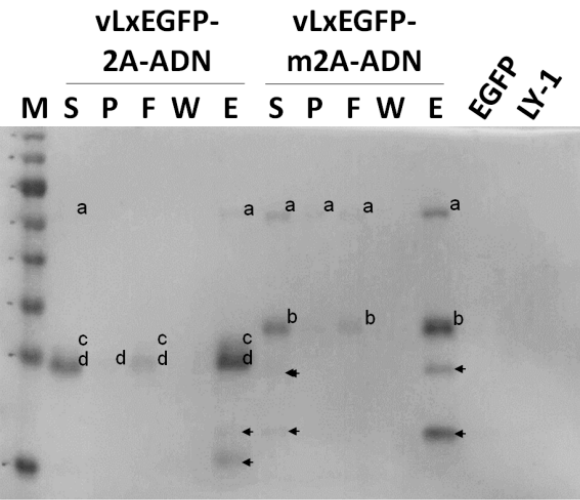

Anti-His Tag

B

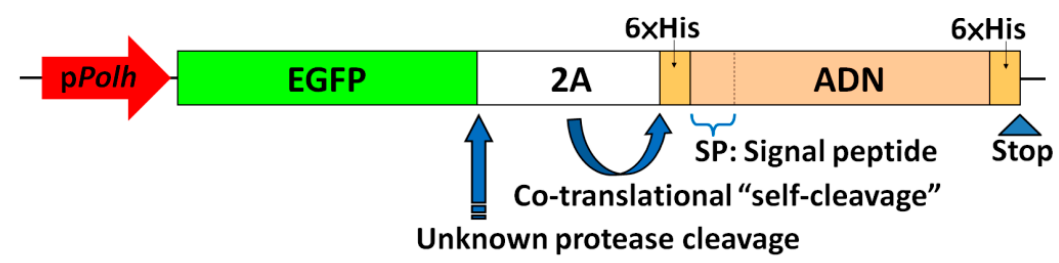

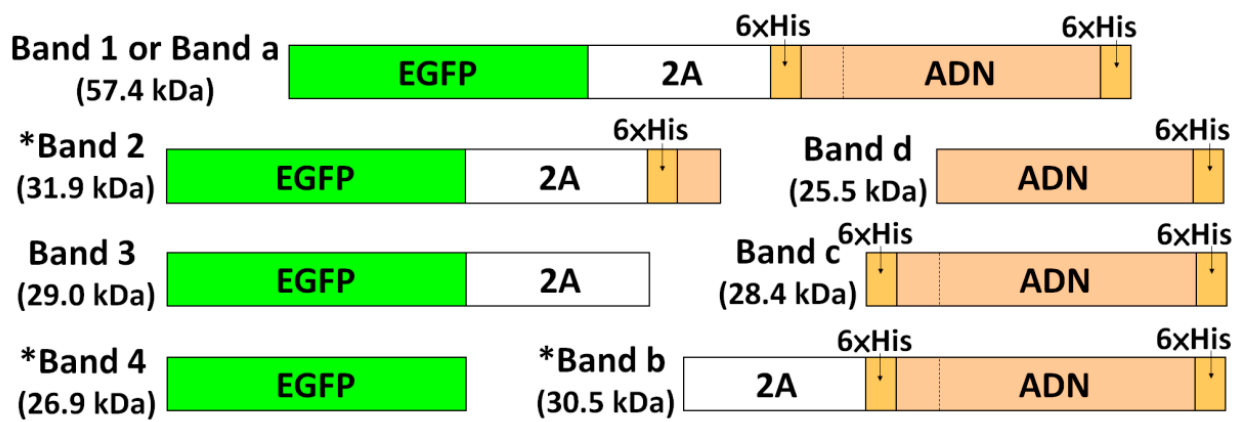

Figure 4. Purification of recombinant proteins and verification of 2A peptide function in NTU-LY cells. (A) Western blot analysis of each fraction from the IMAC purification process with anti-GFP (Clontech) and anti-His tagged protein antibodies (Bioman Scientific Co., LTD). Samples obtained from cell cultures infected with the recombinant virus, vLxEGFP-2A-ADN or vLxEGFP-m2A-ADN are indicated above lanes. Protein bands corresponding to bands 1, 2, 3, 4 and bands a, b, c, d (see below) are indicated. Several low-molecular bands that supposedly resulted from protein degradation are indicated by arrowheads. (B) Diagram of proposed modifications during or after the recombinant protein EGFP-2A-ADN synthesis. In addition to co-translational "self-cleavage" of functional 2A peptide and signal peptide removal, an unknown protease cleavage was proposed between EGFP and the 2A peptide in the modification process. Bands 1, 2, 3, 4 and bands a, b, c, d were subsequently generated. The molecular weights of these bands shown in parentheses were calculated according to their amino acid sequences. Based on the design, the expected products should be band 1 or band a, band 3 , band c, and band $d$, while band 2 , band 4 , and band b are putative products (indicated by $\left.{ }^{*}\right)$. EGFP $=$ proteins obtained from NTU-LY cells infected with a recombinant virus expressing EGFP; $\mathrm{LY}-1$ = proteins obtained from NTU-LY cells; $\mathrm{M}$ = protein marker; $\mathrm{S}=$ supernatant; $\mathrm{P}$ = pellet; $\mathrm{F}=$ fraction sample; $\mathrm{W}=$ sample from washed solution; $\mathrm{E}=$ eluted sample. 


\subsection{Purification of Recombinant Proteins from Culture Medium}

One major band in the elution fraction corresponding to recombinant ADN monomers (about 26-kDa) appeared in the blots for vLxEGFP-2A-ADN (Figure 5A), indicating that the recombinant ADN can be secreted to the culture medium. By contrast, the major band (around 57-kDa) in the elution fraction from vLxEGFP-m2A-ADN carries a mutant 2A that cannot mediate a cleavage between the EGFP and ADN proteins (Figure 5B). To optimize ADN collection and prove that recombinant ADN was secreted (and not by cell lysis), the medium was collected for nine days, and proteins expression (EGFP and ADN) was detected by Western blotting (Figure 5C). The expression of ADN in the medium increasingly accumulated, and the maximum expression of ADN was detected at Day 5. Although EGFP is not a secretory protein, we detected it in the medium at Day 6, suggesting that cell lysis was occurring. Obviously, recombinant ADN was already detected in culture medium before cell lysis, indicating that it was secreted by cells.

(A)

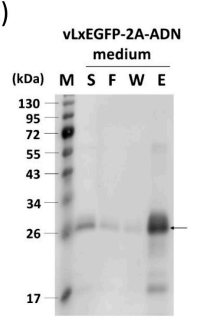

(D)

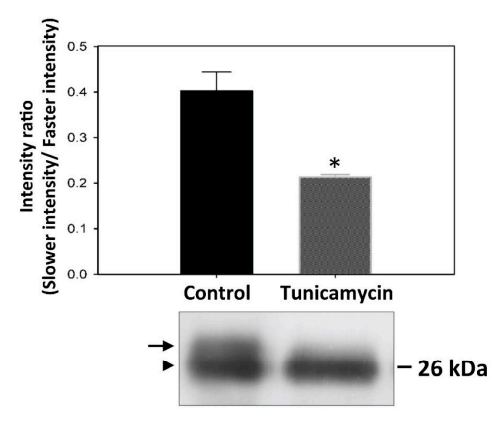

(B)

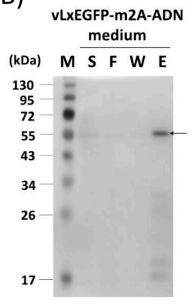

(E)

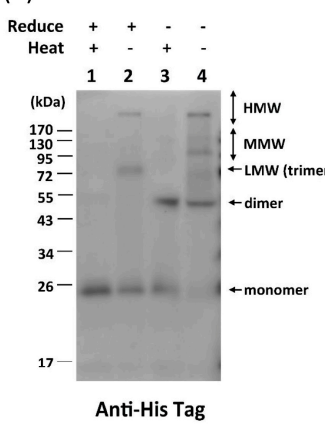

(C)

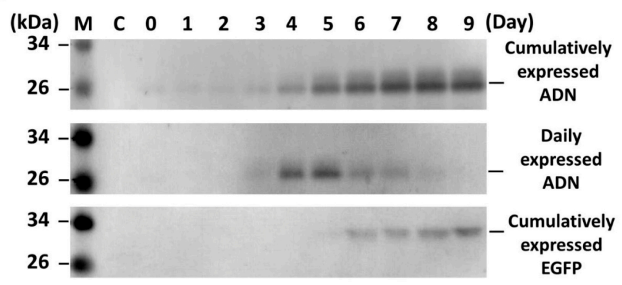

(F)

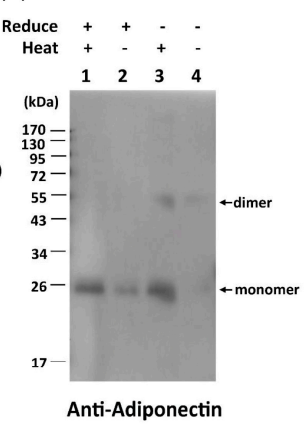

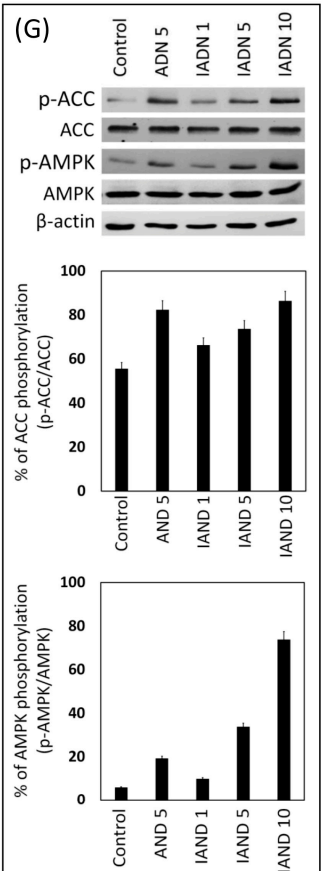

Figure 5. Demonstration of porcine adiponectin (ADN) and EGFP expression by EGFP-2A-based expression system. (A-C) Purification of recombinant proteins from culture medium. The medium obtained from NTU-LY cultures infected with the recombinant virus vLxEGFP-2A-ADN (A) or vLxEGFP-m2A-ADN (B). The major bands representing the purified recombinant proteins in elution fractions are indicated by arrows. (C) Time-course of protein expression in the culture medium. S = supernatant; $\mathrm{F}$ = sample from fraction; $\mathrm{W}=$ sample from washed solution; $\mathrm{E}=$ eluted sample; $\mathrm{C}=$ medium collected from uninfected cultures for the negative control; $\mathrm{M}=$ protein marker; * = significant difference. (D) Glycosylation of recombinant proteins in culture medium. Arrow indicates glycosylated band (slower migrating band), and arrow head indicates non-glycosylated band (faster band). Glycosylated band was decreased more than non-glycosylated band by tunicamycin treatment. (E,F) Multimer separation patterns of ADN expressed in NTU-LY cells by SDS-PAGE under various conditions. Each lane was denoted with "+" (including reducing reagents in sample buffer or heat treatment) or "-" (not including reducing reagents in sample buffer or not heat treatment). HMW= high molecular weight; $\mathrm{MMW}=$ middle molecular weight; LMW = low molecular weight. (G) Effect of recombinant porcine adiponectin on the phosphorylation of acetyl-CoA carboxylase (ACC) and AMP-activated protein kinase (AMPK). Control = cells treated with phosphate buffered saline (PBS, pH 7.4); ADN 5 = cells treated with human recombinant ADN $\left(5 \mu \mathrm{g} \cdot \mathrm{ml}^{-1}\right)$ for positive control; IADN 1, 5, and $10=$ cells treated with insect-expressed porcine recombinant $\operatorname{ADN}\left(1,5\right.$, and $10 \mu \mathrm{g} \cdot \mathrm{ml}^{-1}$, respectively). 


\subsection{Glycosylation of Recombinant Proteins}

Tunicamycin is a known inhibitor of N-linked glycosylation not only in mammalian cells but also in insect cells. In tunicamycin-treated cells, the ratio of slower to faster migrating electrophoretic bands was significantly reduced ( ${ }^{*} p<0.05$ vs. control cells; $0.4 \pm 0.04$ to $0.2 \pm 0.01$, arbitrary unit) (Figure 5D), suggesting that the slower migrating band was glycosylated ADN.

\subsection{Multiuser Separation Patterns of Porcine Adiponectin by SDS-PAGE under Various Conditions}

The expression of recombinant ADN was detected in the medium after the insect cells were infected by vLxGFP-2A-ADN for seven days (Figure 5E,F). The current data show that ADN expression in our system is not only dimers and trimers but also the oligomer of a high molecular weight. For determining the formation of recombinant ADN secreted from insect cells, the media were mixed with reducing buffer or nonreducing buffer, and either heated or not heated. When samples were not reduced or heated, the formation of a range of different multimeric states was present (Figure 4E, lane 4). We observed monomers at $26 \mathrm{kDa}$, dimers at $52 \mathrm{kDa}$, trimers at $72 \mathrm{kDa}$, and other higher-molecular weight multimers up to above $170 \mathrm{kDa}$. Unlike monomers and dimers, larger ADN multimers fail to migrate proportionally to their molecular weights in SDS-PAGE, as they retain certain folded structures under SDS-PAGE conditions (Waki et al. 2003). Interestingly, insect-expressed ADN trimers migrate only slightly slower than dimers, followed by middle molecular weight (MMW) multimers, and then HMW multimers (Figure 5E, lane 4). After the samples were heated, dimer ADN and monomer ADN were produced (Figure 5E,F, lane 3). On the other hand, reduction without heat denaturation converted ADN into HMW, a trimer, and a monomer by anti-His Tag antibody detection, but we only detected a monomer with the anti-ADN antibody (Figure 5E,F, lane 2). The pattern of Western blots by the anti-ADN antibody was similar to the anti-His Tag antibody, indicating that recombinant protein was successfully expressed, while the anti-ADN antibody could not detect the multimeric ADN, suggesting that different origins of the antigen for the monoclonal antibody production might influence the affinity for target protein recognition. Bacterially-expressed ADN lacks posttranslational modifications to be folded correctly and self-associate (Richards et al. 2006). When heated and reduced, the sample was transformed to an approximately $26 \mathrm{kDa}$ monomer, indicating that all of these species are composed of identical monomers, namely, homo-multimers (Figure 5E,F, lane 1).

\subsection{Effect of Recombinant Porcine Adiponectin on the Phosphorylation of AMPK and ACC}

The phosphorylation of AMPK and ACC was investigated in hepatocytes (SK-Hep1) treated with the recombinant proteins. As Figure 5G shows, the insect-produced ADN (IADN) from the new expression system increases the phosphorylation of AMPK and ACC in a dose-dependent manner. More importantly, IADN has a bioactivity similar to that of a commercial recombinant human ADN.

\section{Discussion}

ADN is an adipokine, involved in insulin sensitivity and lower in obese patients [20], which was selected as an example for expression in our system for establishing posttranslational modification. Mammalian cells have only produced commercially available functional recombinant ADN; however, these cells are not ideal for potential biomedical applications partly due to their low yield, high cost, and lack of multimers. The expression of recombinant $\mathrm{ADN}$ by the bacterial culture system produces a complete lack of differentially modified isoforms and fails to form large multimers [18,21]. A facile protein expression system based on the concatenation of the $2 \mathrm{~A}$ peptide element was thought to be a rapid recombinant baculovirus-generating system; moreover, it facilitates the following viral selective progress (Figure 1). The results from this and previous studies [9] have demonstrated that the 2A peptide could be suitable for the co-expression of multiple genes. The advantages of a 2A peptide include the fact that the co-expression of proteins linked by $2 \mathrm{~A}$ is independent of the cell type, and multiple proteins are co-expressed in equimolar amounts from a single transcript mRNA (single ORF) 
under the control of only one promoter, and $2 \mathrm{~A}$ is smaller (54-174 bp) compared to internal ribosome entry site (IRES) elements [22-24]. By introducing a reporter gene, the enhanced green fluorescent protein, EGFP, it allows for easy detection and isolation of the recombinant viruses and also simplifies titer determinations (Figure 3). The results show that a functional 2A peptide mediated an effective separation from EGFP and ADN (Figure 4). Additionally, longer C-terminal 2A peptide extension of the upstream translation product may affect the upstream translation product, including protein conformation and activity [23]. Thus, our ADN sequence was arranged downstream followed by the 2A peptide (Figure 2) to avoid producing an ADN-2A fusion protein in the system. Taken together, the results from this study demonstrated that the newly multiple expression system both accelerated the selection process of recombinant viruses and the determination of expression kinetics and harvest time for ADN production (Figures 1 and 5).

The porcine ADN was expressed with an Escherichia coli system in our pilot study, but an inclusion body was formed (unpublished data). Coincidentally, the previous study had shown that the human recombinant ADN protein expressed by a bacterial culture system completely lacks differentially modified isoforms and fails to form large multimers [25]. The slower migrating form of ADN suggests that porcine ADN may be glycosylated (Figure 5D). The insect cells were treated with tunicamycin, which decreased the intensity ratio of the slower to faster migrating form of ADN. The result indicated that $\mathrm{N}$-linked glycosylation was inhibited (Figure 5D). In addition, the data indicated that the "self-cleavage" process of the 2A peptide maintains the ability for glycosylation. The collagenous region of ADN is glycosylated in vitro with glucosylgalactosyl moieties on four conserved lysines. High molecular weight ADN has a greater degree of glycosylation, leading to a greater stability of higher molecular weight forms [26]. N-linked glycosylation in the anterior sequence is conserved in many mammals (e.g., Sus scrofa, Bos taurus, and Canis lupus) [27], and glycosylation is correlated with the stability of high molecular weight forms. These observations were consistent with our observation that high molecular weight ADN could be detected in the medium (Figure 5E).

Covalent, disulfide bonds are required to form the homo-multimer of ADN. Simple SDS-PAGE under the conditions of nonreducing and non-heat-denaturing separates multimer species of ADN [18]. Reduction and heating in SDS-PAGE break disulfide bonds and ionic interactions, respectively. Disulfide bonds near the N-terminus help the six heterotrimers of $\mathrm{C} 1 \mathrm{q}$ to assemble properly in ADN [28]. The monomer of recombinant ADN was expressed, because the high molecular weight disulfide bonds were dissociated by $\beta$-mercaptoehanol (Figure 5E,F). The dragging pattern of protein migration in the gel in lane 4 (Figure 5E,F) indicated nondenature of the covalently linked disulfide bond and ionic interaction among the adiponectin multimers. After heating with the ADN, major expression of the dimer and monomer was detected, which assumed that not all the adiponectin dimer was linked by disulfide-bonds (Figure $5 \mathrm{E}, \mathrm{F}$ ). An insect ovarian cell culture system produces monomers, dimers, and trimers but not higher molecular weight forms of ADN [29]. On the other hand, our insect cell system produced higher molecular weight forms and dimers of ADN (Figure 5E,F). Moreover, the commercial BacPAK ${ }^{\mathrm{TM}}$ baculovirus expression system (BD Biosciences), which is expressed in insect H5 cells (Invitrogen, Karlsruhe, Germany), can also generate functional adiponectin [30]. However, the formation of $\mathrm{ADN}$ in $\mathrm{BaCPAK}^{\mathrm{TM}}$ is most likely low-molecular weight $\mathrm{ADN}$. The active forms of ADN need posttranslational modification to form higher molecular weight forms [28,31,32], while this modification in insect cells may be destroyed by the cytopathogenic effects of the baculovirus vectors during the very-late phase of infection. We assumed that our system with detectable expression kinetics (Figures 3 and $5 \mathrm{C}$ ) and a long-term expression capacity (e.g., the maximum expression of ADN was detected at Day 5) is more suitable for producing high molecular weight ADN (Figure 5E). By contrast, in a conventional AcMNPV system, the productivity of infected insect cells peaks before $72 \mathrm{~h}$ post-infection while declining dramatically [33]. High molecular weight ADN activates AMPK in the liver [18,34], and the regulation of AMPK by ADN is a critical determinant of energy metabolism [35]. The phosphorylation of the downstream proteins, AMPK and ACC in SK-Hep1, indicated that functional ADN was produced in our system (Figure 5G). This biological activity is 
consistent with that observed with the recombinant protein produced with a mouse myeloma cell line (NS0) [30]. Interestingly, different ADN multimers activate different signal transduction pathways, while the ADN multimer species can be changed by the protein synthesis process of different eukaryotic donor cells because of the difference of the posttranslational modification system and variation of amino acids sequences within a eukaryote or even among animal species [21,28]. Mice expressing porcine ADN receptors are resistant to the development of weight gain, hepatosteatosis, and insulin resistance [36]. To address the mechanism of these porcine receptors in adipose tissue, our recombinant porcine ADN will be a valuable tool.

\section{Conclusions}

In this study, we demonstrated that incorporating an EGFP and a viral-derived "self-cleaving" 2A peptide in a baculoviral vector facilitated the establishment of a BEVS, increasing the efficiency of target protein expression. Furthermore, the target multimers of proteins with biological activity were formed by posttranslational modification in our system. This functional ADN with biological activity suggested that the system could be applied as a valuable tool to produce recombinant proteins for medicinal purposes. Moreover, tracking the toxic protein easily in vivo could be utilized in our system by co-expressing a toxic protein (e.g., scorpion) with the EGFP recombinant virus. Therefore, we believe that the results and the concept we provided can be utilized in other combinations of baculovirus/insect cell expression systems for various applications.

Supplementary Materials: The following are available online at http://www.mdpi.com/2227-9717/7/5/291/s1.

Author Contributions: Conceptualization, C.-Y.W.; methodology, C.-Y.W. and C.-W.H.; validation, C.-Y.W., C.-W.H. and Y.-S.N.; formal analysis, C.-Y.W. and C.-W.H.; investigation, C.-Y.W., C.-W.H. and P.-Y.C.; resources, C.-Y.W., S.-T.D. and C.-H.W.; writing-original draft preparation, C.-Y.W., C.-W.H. and Y.-S.N.; writing-review and editing, C.-Y.W., C.W.H. and Y.-S.N.; visualization, C.-Y.W. and Y.-S.N.; supervision, C.-H.W. and S.-T.D.; project administration, C.-Y.W. and S.-T.D.; funding acquisition, C.-Y.W. and S.-T.D.

Funding: This research was funded by the grant NSC 100-2321-B-002-070-MY2 from the National Science Council of Taiwan and the grant No.100AS-1.2.2-S-a2(1) from the Council of Agriculture, Executive Yuan, Republic of China.

Acknowledgments: This work was supported by the grant NSC 100-2321-B-002-070-MY2 from the National Science Council of Taiwan and the grant No.100AS-1.2.2-S-a2(1) from the Council of Agriculture, Executive Yuan, Republic of China.

Conflicts of Interest: All authors declare that they have no conflict of interest.

Compliance with Ethical Standards: The Institutional Animal Care and Use Committee of National Taiwan University approved the experimental protocol (NTU-96-EL-50). All animal experiments were performed according to regulations approved by the Animal Ethical Committee of National Taiwan University.

\section{References}

1. Murhammer, D.W. Baculovirus and Insect Cell Expression Protocols; Methods in Moleculer Biology; Humana Press: New York, NY, USA, 2007.

2. Smith, G.E.; Summers, M.D.; Fraser, M.J. Production of human beta interferon in insect cells infected with a baculovirus expression vector. Mol. Cell. Biol. 1983, 3, 2156-2165. [CrossRef]

3. Miller, L.K. Baculoviruses as gene expression vectors. Annu. Rev. Microbiol. 1988, 42, 177-199. [CrossRef] [PubMed]

4. Luckow, V.A.; Summers, M.D. Trends in the development of baculovirus expression vectors. Nat. Biotechnol. 1988, 6, 47-55. [CrossRef]

5. Steele, K.H.; Stone, B.J.; Franklin, K.M.; Fath-Goodin, A.; Zhang, X.; Jiang, H.; Webb, B.A.; Geisler, C. Improving the baculovirus expression vector system with vankyrin-enhanced technology. Biotechnol. Prog. 2017, 33, 1496-1507. [CrossRef]

6. Wang, J.; Hou, D.; Wang, Q.; Kuang, W.; Zhang, L.; Li, J.; Shen, S.; Deng, F.; Wang, H.; Hu, Z.; et al. Genome analysis of a novel Group I alphabaculovirus obtained from Oxyplax ochracea. PLoS ONE 2018, 13. [CrossRef] 
7. Sussman, D.J. 24-hour assay for estimating the titer of beta-galactosidase-expressing baculovirus. Biotechniques 1995, 18, 50-51.

8. de Felipe, P.; Ryan, M.D. Targeting of proteins derived from self-processing polyproteins containing multiple signal sequences. Traffic 2004, 5, 616-626. [CrossRef] [PubMed]

9. de Felipe, P.; Luke, G.A.; Hughes, L.E.; Gani, D.; Halpin, C.; Ryan, M.D. E unum pluribus: Multiple proteins from a self-processing polyprotein. Trends Biotechnol. 2006, 24, 68-75. [CrossRef]

10. Mansouri, M.; Bellon-Echeverria, I.; Rizk, A.; Ehsaei, Z.; Cianciolo Cosentino, C.; Silva, C.S.; Xie, Y.; Boyce, F.M.; Davis, M.W.; Neuhauss, S.C.; et al. Highly efficient baculovirus-mediated multigene delivery in primary cells. Nat. Commun. 2016, 7. [CrossRef] [PubMed]

11. Liu, Z.; Chen, O.; Wall, J.B.J.; Zheng, M.; Zhou, Y.; Wang, L.; Ruth Vaseghi, H.; Qian, L.; Liu, J. Systematic comparison of 2A peptides for cloning multi-genes in a polycistronic vector. Sci. Rep. 2017, 7. [CrossRef] [PubMed]

12. Kim, J.H.; Lee, S.R.; Li, L.H.; Park, H.J.; Park, J.H.; Lee, K.Y.; Kim, M.K.; Shin, B.A.; Choi, S.Y. High cleavage efficiency of a 2A peptide derived from porcine teschovirus-1 in human cell lines, zebrafish and mice. PLoS ONE 2011, 6, e18556. [CrossRef]

13. Wang, Y.; Wang, F.; Wang, R.; Zhao, P.; Xia, Q. 2A self-cleaving peptide-based multi-gene expression system in the silkworm Bombyx mori. Sci. Rep. 2015, 5, 16273. [CrossRef]

14. Wang, Y.; Wang, F.; Xu, S.; Wang, R.; Chen, W.; Hou, K.; Tian, C.; Wang, F.; Zhao, P.; Xia, Q. Optimization of a 2A self-cleaving peptide-based multigene expression system for efficient expression of upstream and downstream genes in silkworm. Mol. Genet. Genom. 2019. [CrossRef] [PubMed]

15. Wu, C.Y.; Wang, C.H. New cell lines from Lymantria xylina (Lepidoptera: Lymantriidae): Characterization and susceptibility to baculoviruses. J. Invertebr. Pathol. 2006, 93, 186-191. [CrossRef]

16. Hink, W.; Strauss, E. Growth of the Trichoplusia ni (TN-368) cell line in suspension culture. Int. Conf. Invertebr. Tissue Culture Appl. Med. Biol. Agric. 1976. [CrossRef]

17. Nai, Y.S.; Wu, C.Y.; Wang, T.C.; Chen, Y.R.; Lau, W.H.; Lo, C.F.; Tsai, M.F.; Wang, C.H. Genomic sequencing and analyses of Lymantria xylina multiple nucleopolyhedrovirus. BMC Genomics 2010, 11. [CrossRef]

18. Waki, H.; Yamauchi, T.; Kamon, J.; Ito, Y.; Uchida, S.; Kita, S.; Hara, K.; Hada, Y.; Vasseur, F.; Froguel, P.; et al. Impaired multimerization of human adiponectin mutants associated with diabetes. Molecular structure and multimer formation of adiponectin. J. Biol. Chem. 2003, 278, 40352-40363. [CrossRef] [PubMed]

19. Petersen, T.N.; Brunak, S.; von Heijne, G.; Nielsen, H. SignalP 4.0: Discriminating signal peptides from transmembrane regions. Nat. Methods. 2011, 8, 785-786. [CrossRef] [PubMed]

20. Trujillo, M.E.; Scherer, P.E. Adiponectin-journey from an adipocyte secretory protein to biomarker of the metabolic syndrome. J. Intern. Med. 2005, 257, 167-175. [CrossRef]

21. Richards, A.A.; Stephens, T.; Charlton, H.K.; Jones, A.; Macdonald, G.A.; Prins, J.B.; Whitehead, J.P. Adiponectin multimerization is dependent on conserved lysines in the collagenous domain: evidence for regulation of multimerization by alterations in posttranslational modifications. Mol. Endocrinol. 2006, 20, 1673-1687. [CrossRef]

22. Osborn, M.J.; Panoskaltsis-Mortari, A.; McElmurry, R.T.; Bell, S.K.; Vignali, D.A.; Ryan, M.D.; Wilber, A.C.; McIvor, R.S.; Tolar, J.; Blazar, B.R. A picornaviral 2A-like sequence-based tricistronic vector allowing for high-level therapeutic gene expression coupled to a dual-reporter system. Mol. Ther. 2005, 12, 569-574. [CrossRef] [PubMed]

23. Minskaia, E.; Nicholson, J.; Ryan, M.D. Optimisation of the foot-and-mouth disease virus 2A co-expression system for biomedical applications. BMC Biotechnol. 2013, 13. [CrossRef] [PubMed]

24. Ibrahimi, A.; Vande Velde, G.; Reumers, V.; Toelen, J.; Thiry, I.; Vandeputte, C.; Vets, S.; Deroose, C.; Bormans, G.; Baekelandt, V.; et al. Highly efficient multicistronic lentiviral vectors with peptide 2 A sequences. Hum. Gene. Ther. 2009, 20, 845-860. [CrossRef] [PubMed]

25. Hu, X.B.; Zhang, Y.J.; Zhang, H.T.; Yang, S.L.; Gong, Y. Cloning and expression of adiponectin and its globular domain, and measurement of the biological activity in vivo. Sheng Wu Hua Xue Yu Sheng Wu Wu Li Xue Bao (Shanghai) 2003, 35, 1023-1028.

26. Peake, P.W.; Hughes, J.T.; Shen, Y.; Charlesworth, J.A. Glycosylation of human adiponectin affects its conformation and stability. J. Mol. Endocrinol. 2007, 39, 45-52. [CrossRef] [PubMed]

27. Tanaka, M.; Fukuhara, A.; Shimomura, I. N-linked glycosylation of mouse adiponectin. Horm. Metab. Res. 2011, 43, 545-550. [CrossRef] [PubMed] 
28. Tsao, T.S.; Tomas, E.; Murrey, H.E.; Hug, C.; Lee, D.H.; Ruderman, N.B.; Heuser, J.E.; Lodish, H.F. Role of disulfide bonds in Acrp30/adiponectin structure and signaling specificity. Different oligomers activate different signal transduction pathways. J. Biol. Chem. 2003, 278, 50810-50817. [CrossRef]

29. Yuh, I.S.; Sheffield, L.G. Adiponectin gene cloning and its expression in insect cell expression system. Reprod. Dev. Biol. 2012, 36, 193-198.

30. Neumeier, M.; Weigert, J.; Schaffler, A.; Wehrwein, G.; Muller-Ladner, U.; Scholmerich, J.; Wrede, C.; Buechler, C. Different effects of adiponectin isoforms in human monocytic cells. J. Leukoc. Biol. 2006, 79, 803-808. [CrossRef] [PubMed]

31. Yamauchi, T.; Kamon, J.; Waki, H.; Terauchi, Y.; Kubota, N.; Hara, K.; Mori, Y.; Ide, T.; Murakami, K.; Tsuboyama-Kasaoka, N.; et al. The fat-derived hormone adiponectin reverses insulin resistance associated with both lipoatrophy and obesity. Nat. Med. 2001, 7, 941-946. [CrossRef] [PubMed]

32. Wang, Y.; Xu, A.; Knight, C.; Xu, L.Y.; Cooper, G.J. Hydroxylation and glycosylation of the four conserved lysine residues in the collagenous domain of adiponectin. Potential role in the modulation of its insulin-sensitizing activity. J. Biol. Chem. 2002, 277, 19521-19529. [CrossRef]

33. Gomez-Sebastian, S.; Lopez-Vidal, J.; Escribano, J.M. Significant productivity improvement of the baculovirus expression vector system by engineering a novel expression cassette. PLoS ONE 2014, 9, e96562. [CrossRef]

34. Wang, Y.; Lam, K.S.; Yau, M.H.; Xu, A. Post-translational modifications of adiponectin: Mechanisms and functional implications. Biochem. J. 2008, 409, 623-633. [CrossRef] [PubMed]

35. Spurlock, M.E.; Gabler, N.K. The development of porcine models of obesity and the metabolic syndrome. J. Nutr. 2008, 138, 397-402. [CrossRef] [PubMed]

36. Liu, B.H.; Lin, Y.Y.; Wang, Y.C.; Huang, C.W.; Chen, C.C.; Wu, S.C.; Mersmann, H.J.; Cheng, W.T.; Ding, S.T. Porcine adiponectin receptor 1 transgene resists high-fat/sucrose diet-induced weight gain, hepatosteatosis and insulin resistance in mice. Exp. Anim. 2013, 62, 347-360. [CrossRef] [PubMed]

(C) 2019 by the authors. Licensee MDPI, Basel, Switzerland. This article is an open access article distributed under the terms and conditions of the Creative Commons Attribution (CC BY) license (http://creativecommons.org/licenses/by/4.0/). 